TITLE:

\title{
Tiny waveform receiver with a dedicated system chip for observing plasma waves in space
}

\section{AUTHOR(S):}

Fukuhara, H; Kojima, H; Ishii, H; Okada, S; Yamakawa, $\mathrm{H}$

\section{CITATION:}

Fukuhara, $H$...[et al]. Tiny waveform receiver with a dedicated system chip for observing plasma waves in space. Measurement Science and Technology 2012, 23(10): 105903.

ISSUE DATE:

2012-10

URL:

http://hdl.handle.net/2433/159518

\section{RIGHT:}

(c) 2012 IOP Publishing Ltd; This is not the published version. Please cite only the published version.; この論文は出版社版でありません。引用の 際には出版社版をご確認ご利用ください。 


\title{
Tiny Waveform Receiver with a Dedicated System Chip for Observing Plasma Waves in Space
}

\author{
H Fukuhara ${ }^{1}$, H Kojima ${ }^{1}$, H Ishii ${ }^{1}$, S Okada ${ }^{1}$, and H Yamakawa ${ }^{1}$ \\ Research Institute for Sustainable Humanosphere, Kyoto University, Gokasho, Uji, \\ Kyoto, 611-0011, Japan \\ E-mail: fukuhara@rish.kyoto-u.ac.jp
}

\begin{abstract}
Plasma waves are important observational targets for scientific satellite missions to investigate electromagnetic phenomena that occur in space. For future scientific missions, reduction in the resource requirements of plasma wave receivers without loss of performance is important. The present paper introduces a miniaturized on-board instrument for the observation of plasma waves using analogue applicationspecific integrated circuit (ASIC) techniques. The developed ASIC functions as a system chip to filter and amplify signals detected by plasma wave sensors. Miniaturization of the analogue circuit using the ASIC leads to the realization of a tiny plasma wave receiver. The overall size of the developed plasma wave receiver circuit board is less than $1 / 20$ that of a conventional receiver used in previous scientific missions. The power consumption of the system chip and the plasma wave receiver is 165 and $525 \mathrm{~mW}$, respectively.
\end{abstract}




\section{Introduction}

Space is filled with very subtle plasmas that are essentially collisionless. The kinetic energies of plasmas are exchanged not through collision, but through field quantities, such as electric and magnetic fields, i.e., plasma waves. Plasma waves are regarded as a type of radio wave involving plasma. Since plasma is a dispersive medium, many modes exist that cover a wide frequency range of up to a few tens of megahertz.

A number of scientific spacecraft have been launched to investigate electromagnetic environments in space. Small dipole antennas, loop antennas, and search coils are used for sensors on board these spacecraft. The signals received by the sensors are transmitted to the ground after on-board signal processing, and plasma waves are typically observed as frequency spectra. Matsumoto et al. (1994a) realized a breakthrough in plasma wave observation through the use of a digital waveform receiver on board the GEOTAIL spacecraft [1]. The obtained waveform contains not only the amplitude, but also the phase of the plasma waves. Consequently, this waveform receiver contributed to a number of scientific discoveries. For example, Matsumoto et al. (1994b) discovered solitary electrostatic waves by analyzing waveform data from GEOTAIL [2]. Consequently, the importance of waveform observation has increased and such observation has currently become common in scientific missions $[3,4,5,6]$.

Recently, requirements to reduce the weight budgets for on-board instruments are more stringent than ever, due to growing scientific demands for multi-spacecraft and planetary missions. The digital components of on-board instruments can be easily downsized by using highly integrated circuits developed with recent semiconductor technologies. However, analogue components are much more difficult to miniaturize. Plasma waves exist in a wide frequency band with various intensities, as shown in Figure 1 ; therefore, the analogue components of the plasma wave receiver must have low noise, high sensitivity, and a wide dynamic range. Thus, the use of highly reliable parts that meet military specifications, but are less integrated, has been key in the development of the analogue components for these instruments.

A low-noise amplifier with programmable gain was developed as a dedicated system chip, an application-specific integrated circuit (ASIC), for plasma wave observations [7]. The amplifier was designed to be radiation tolerant, in addition to having low noise of $100 \mathrm{nV} / \sqrt{\mathrm{Hz}}$ at $100 \mathrm{~Hz}$, using the National Semiconductor SiGe 0.25- $\mu \mathrm{m}$ BiCMOS process. Robustness toward total ionizing dose and single event effects were confirmed. Fukuhara et al. (2011) developed an ASIC that included not only amplifiers, but also filters for the waveform receiver, using the TSMC 0.25- $\mu \mathrm{m}$ CMOS process [8]. These ASICs demonstrated that the analogue components of the waveform receiver could be significantly downsized. An ASIC is very small and easily mass-produced, so applying it to on-board instruments is advantageous for multi-spacecraft missions involving small, micro-, or nano-satellites.

The objective of the present paper is to demonstrate the overall performance of the developed system chip and to introduce a tiny circuit board for a waveform receiver. 


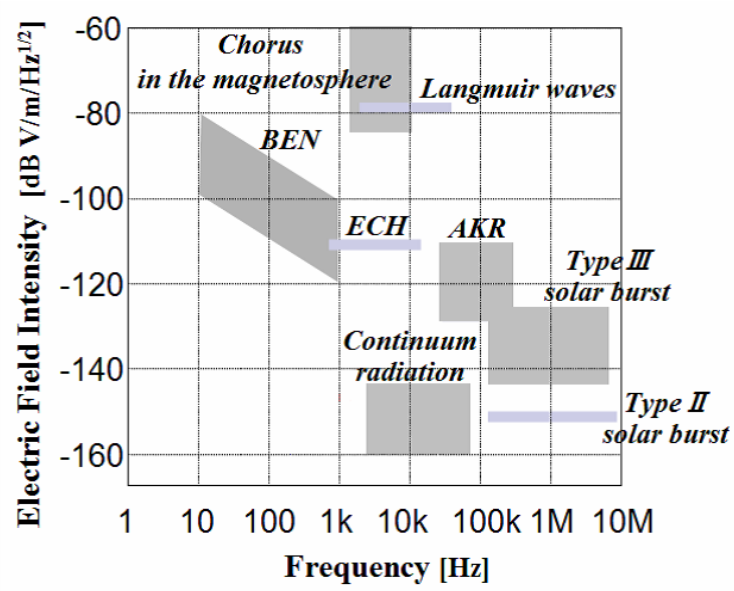

Figure 1. Intensities and frequency bands of typical plasma waves in the terrestrial magnetosphere.

The current ASIC was fabricated using the TSMC 0.25- $\mu \mathrm{m}$ CMOS process. The size of the waveform receiver was successfully reduced to $45 \times 50 \mathrm{~mm}^{2}$, which is approximately half the size of a business card. The sensitivity and dynamic range are acceptable for scientific observations, and as such, the miniaturized plasma wave receiver is expected to be used for novel applications [9].

The remainder of the paper is organized as follows. In Section 2, the design of the waveform receiver is summarized. The details and performance of the system chip and the circuit board are presented in Section 3, and conclusions are given in Section 4.

\section{Circuit Design Summary}

\subsection{Requirements}

Plasma waves exist in a wide frequency band and a very wide signal dynamic range, as shown in Figure 1. The upper frequency limit of the waveform receiver is typically a few hundred kilohertz and is determined by considering the nature of the physical target phenomena, in addition to the power budget and telemetry capacity. A waveform receiver with an upper frequency of a few hundred kilohertz covers the local electron plasma frequency in the outer magnetosphere and the local ion plasma frequency in the ionosphere.

The waveform receiver acts as an analogue front-end system with a wide frequency band. The first requirement of the receiver is that it must have low noise levels. The ability to amplify weak signals is also necessary. Low-noise band-limiting low-pass filters (LPF) are required in front of the amplifier to avoid saturation. In addition, maintaining a wide dynamic range for the receiver requires gain control of the amplifier.

For sample-and-hold and analogue-to-digital (A/D) conversion, anti-aliasing filters must be placed after the amplifiers. Moreover, six channels are required to observe the 
full set of electric and magnetic fields.

The sensitivity of the receiver is required to be $-120 \mathrm{dBV} / \sqrt{\mathrm{Hz}}$ at $1 \mathrm{kHz}$, and $-140 \mathrm{dBV} / \sqrt{\mathrm{Hz}}$ at $100 \mathrm{kHz}$. These values are comparable to the typical output voltagenoise density of a preamplifier. The small sensor node proposed by Kojima et al. (2010) requires a very small electric field sensor [9], although conventional scientific spacecraft generally have an electric field sensor with an effective length of greater than $10 \mathrm{~m}$. Assuming that the effective length of the small dipole antenna for the electric field sensor is at least $1 \mathrm{~m}$, the sensitivity requirements enable the receiver to observe several types of plasma waves, such as electron cyclotron harmonics (ECH), auroral kilometric radiation, and type-III solar bursts. The receiver noise must be sufficiently low to satisfy the sensitivity requirements with the small size of the electric field sensor.

\subsection{Specifications}

The specifications of the tiny waveform receiver are summarized herein and compared with the NOZOMI (previous Japanese Mars explorer mission) low-frequency analyzer (LFA) in Table 1. The LFA is an on-board waveform receiver [10] designed for operation under conditions similar to the terrestrial magnetosphere.

Table 1. Specifications of the waveform receiver. (*: Exclusive power consumption of the NOZOMI LFA is unstated.)

\begin{tabular}{lcc}
\hline & Present Design & NOZOMI LFA \\
\hline Electric Field & Three components & Two components \\
Magnetic Field & Three components & None \\
Upper Limit Freq. & $100 \mathrm{kHz}$ & $32 \mathrm{kHz}$ \\
Sampling Frequency & $400 \mathrm{kHz}$ & $100 \mathrm{kHz}$ \\
Gain & $0,20,40 \mathrm{~dB}$ & $0,20 \mathrm{~dB}$ \\
Dynamic Range & $78 \mathrm{~dB}(14 \mathrm{bit})$ & $90 \mathrm{~dB}(16 \mathrm{bit})$ \\
Sensitivity & $-120 \mathrm{dBV} / \sqrt{\mathrm{Hz}} @ 1 \mathrm{kHz}$ & $-117 \mathrm{dBV} @ 1 \mathrm{kHz}$ \\
Size of Analog Part & $-140 \mathrm{dBV} / \sqrt{\mathrm{Hz}} @ 100 \mathrm{kHz}$ & $-111 \mathrm{dBV} @ 32 \mathrm{kHz}$ \\
Power Consumption & $45 \times 50 \mathrm{~mm}{ }^{2}$ & $1 / 3 \mathrm{of} \mathrm{A4-size}$ \\
\hline
\end{tabular}

The specifications of the tiny waveform receiver are generally superior to those of the LFA in most respects. The six observation channels enable the analysis of a complete set of electromagnetic fields. Only the dynamic range is lower than that of the NOZOMI LFA. Assuming that a monolithic wave is received, the bandwidth can be set to $1 \mathrm{~Hz}$. The sensitivity of the present design is therefore expected to provide better performance than the previous instrument. In addition, the power consumption should be lower than $800 \mathrm{~mW}$. 


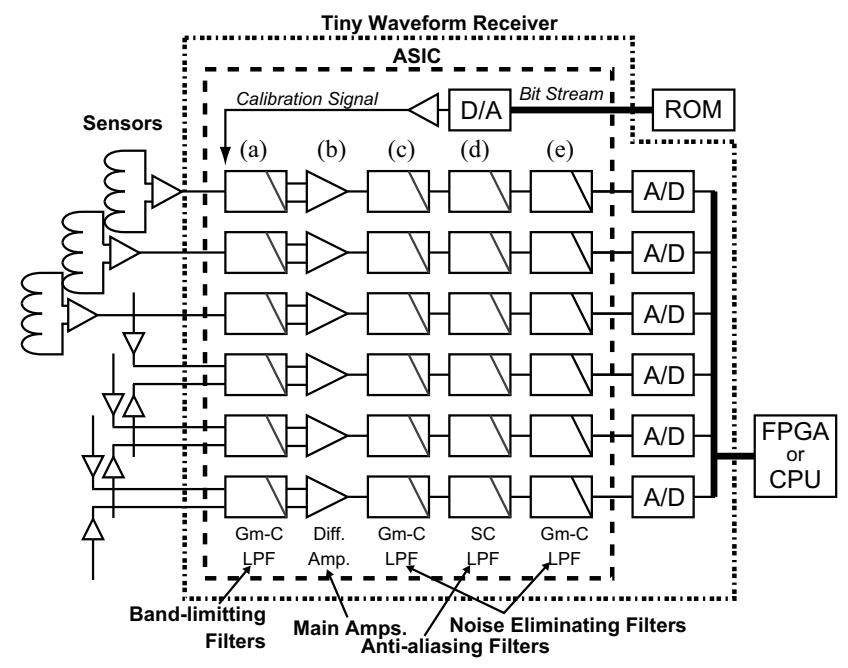

Figure 2. Block diagram of the system chip for the waveform receiver.

The details of the waveform receiver with the system chip and peripheral circuitries are described in the following sections.

\section{Detailed Description of the Waveform Receiver}

\subsection{System Chip}

A block diagram of the system chip is shown in Figure 2. The system chip includes the analogue circuitry, except for the preamplifiers and A/D converters.

Figure 2 shows that four filters are included in a single channel. For integrated circuits, two types of analog filters can be used: a continuous-time filter such as a Gm$\mathrm{C}$ filter, or a discrete-time filter such as a switched capacitor (SC) filter. The filter response of a $\mathrm{Gm}-\mathrm{C}$ filter is determined by the capacitance and transconductance of an operational transconductance amplifier (OTA). The transconductance of the OTA can be adjusted using its bias current. Gm-C filters have relatively low-noise characteristics and do not require an external signal or a local oscillator. However, they are subject to a large amount of temperature drift and considerable manufacturing variation, which typically necessitates compensation circuits. The SC filter is a discrete-time filter, in which a clock signal is used to switch connections between capacitors and OP-amps. The characteristics of the SC filter are determined by capacitance ratios, resulting in acceptable manufacturing accuracy. Therefore, SC filters can be adopted as filters that must be manufactured with high accuracy. However, an external signal or local oscillator is necessary for switching, and switching generates noise in the SC filter. Switching also causes aliasing due to the sampling of unwanted signals in the frequency band around half that of the switching clock frequency. Therefore, noise eliminating filters are combined with the SC filter.

Thus, because of the noise requirements, the band-limiting filter ((a) in Figure 2) 
employed is a Gm-C filter. The temperature drift is compensated for by using a current source with piecewise linear temperature characteristics. When the temperature of the system chip increases, the bias currents of the OTAs are also increased by inputting an increased compensation current from the current source. The temperature drift of the gain is small (within $1 \mathrm{~dB}$ ); therefore, the current source compensates the temperature dependence of the cut-off frequency for the $\mathrm{Gm}-\mathrm{C}$ filter within $6 \%$, from -30 to $60{ }^{\circ} \mathrm{C}$ [11].

The SC filter is suitable for use as an anti-aliasing filter ((d) in Figure 2), because the anti-aliasing filter should have an accurate frequency response. A sixth-order Chebyshev type filter is adopted to realize a cut-off frequency of $100 \mathrm{kHz}$ and a $78 \mathrm{~dB}$ reduction in the gain at $300 \mathrm{kHz}$. The clock frequency of the SC filter is set to $10 \mathrm{MHz}$. Two Gm-C filters ((c) and (e) in Figure 2) are inserted into the front and rear of the SC filter to eliminate noise. The amplifier ((b) in Figure 2) has three gain steps: 0, 20, and $40 \mathrm{~dB}$. The frequency responses of these filters and the amplifier are shown in Figure 3.

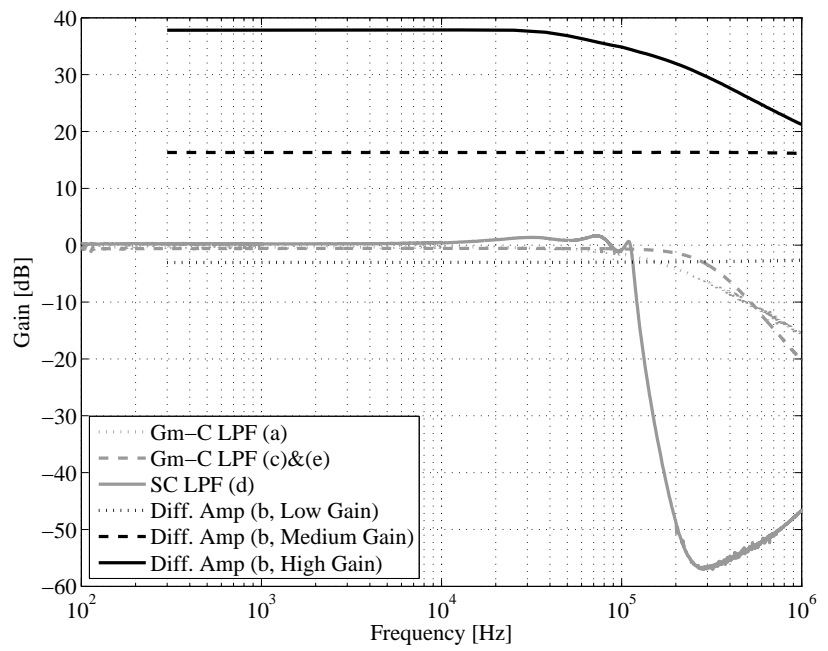

Figure 3. Gain frequency response of each component in the system chip

The digital-to-analogue (D/A) converter in Figure 2 is included for on-board calibration. An antenna in a plasma has different characteristics from that in a vacuum. In addition, the antenna impedance is strongly dependent on the plasma density and the temperature. Therefore, on-board calibration is necessary to obtain absolute quantification of the electric field components of the plasma waves. The calibration system is typically implemented close to the waveform receiver. An analogue calibration signal is obtained by appropriate setting of the input for the D/A converter. It is assumed that the calibration signal is a set of several monolithic waves or a chirp signal from several hundred hertz to $100 \mathrm{kHz}$. The waveform data of the calibration signal are digitally stored in a flash memory and periodically read out for D/A conversion. The calibration signal is fed to the waveform receiver after D/A conversion. The D/A converter is implemented with binary-weighted and thermometer-code current mirrors. 


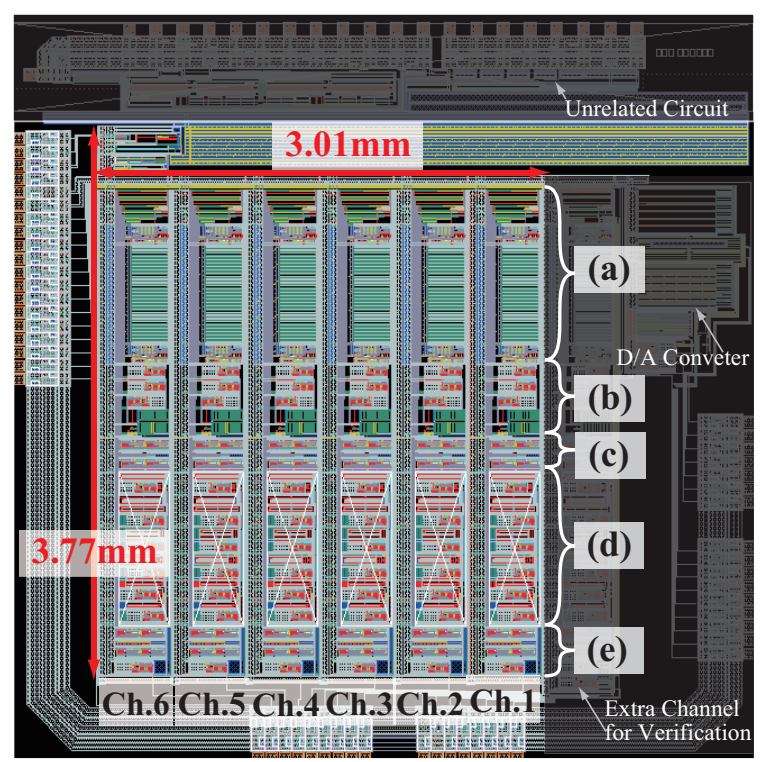

Figure 4. Layout design of the system chip.

The resolution of the $\mathrm{D} / \mathrm{A}$ converter is 8 bits, the upper 4 bits of which are used for the thermometer code and the lower 4 bits of which are used for the binary-weighted code. Correct operation of the $\mathrm{D} / \mathrm{A}$ converter has been confirmed with a differential nonlinearity of $1 \mathrm{~dB}$.

The layout of the system chip is shown in Figure 4, in which the six channels appear stacked horizontally, and the dimensions are $3.77 \times 3.01 \mathrm{~mm}^{2}$. The manufactured chip is contained in an 80-pin quad flat package. The bonding wires connect to the pins through electrostatic discharge protection pads inside the chip. The dimensions of the die and the package are $5 \times 5 \mathrm{~mm}^{2}$ and $15 \times 15 \mathrm{~mm}^{2}$, respectively.

Figure 5 shows the typical gain and phase frequency response performance of the system chip for each gain-setting. Within the pass band, gains of $-2.38,17.1$, and $36.1 \mathrm{~dB}$ are observed for the each gain-setting. The differences in these gains from the designed gains are due to the output conductance error of the internal OP-amps. The attenuation of the designed filter is insufficient in the frequency range 200 to $300 \mathrm{kHz}$. This is due to the existence of a zero point in the transfer function, and will be improved in the next version. The pass-band gain and dynamic range at 1 and $100 \mathrm{kHz}$ are shown in Table 2 with the corresponding gain in the attenuation band that causes aliasing. The effective number of bits of the A/D converter decreases to 10 for the low-gain setting. The medium- and high-gain settings provide a dynamic range corresponding to 14-bit resolution of the $\mathrm{A} / \mathrm{D}$ converter.

\subsection{Peripheral Circuitries}

A block diagram of the overall waveform receiver is shown in Figure $6 . \mathrm{A}+5 \mathrm{~V}$ power supply, the ground level, and six differential pairs of input signals are fed to the waveform 

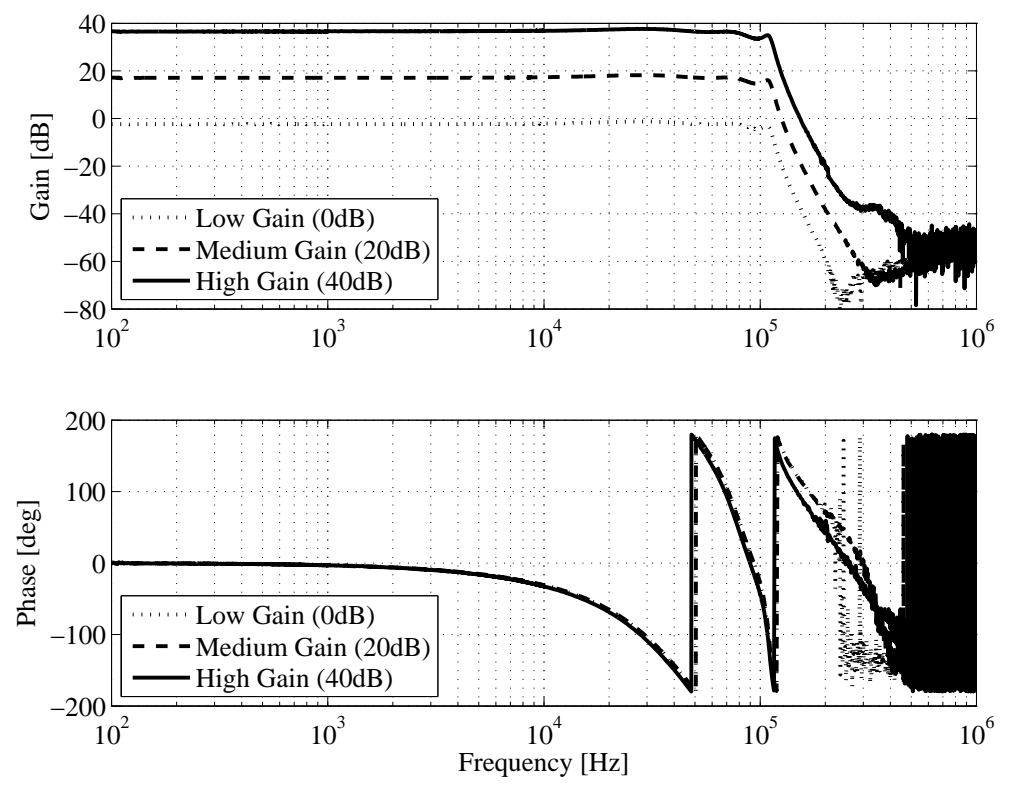

Figure 5. Gains and phases for the frequency response of the chip on the waveform receiver module. The pass band gain can be set to three different values: low $(0 \mathrm{~dB})$, medium $(20 \mathrm{~dB})$, and high $(40 \mathrm{~dB})$ gain.

Table 2. Gain and dynamic range at 1 and $100 \mathrm{kHz}$ with corresponding gain that causes aliasing.

\begin{tabular}{lccc}
\hline Gain Setting & Low & Medium & High \\
\hline Gain @ 1 kHz [dB] & -2.38 & 17.1 & 36.1 \\
Gain @ 399-401 kHz [dB] & -60 & -62 & -39 \\
Dynamic Range @ 1 kHz [dB] & 62 & 79 & 75 \\
\hline Gain @ 100 kHz [dB] & -4.16 & 14.9 & 33.7 \\
Gain @ 300/500 kHz [dB] & $-64 /-58$ & $-62 /-62$ & $-36 /-47$ \\
Dynamic Range @ $100 \mathrm{kHz}[\mathrm{dB}]$ & 54 & 75 & 70 \\
\hline
\end{tabular}

receiver. Common-mode noise on the power line is rejected in the common-mode choke coil. The 3.3 and $1.6 \mathrm{~V}$ power supplies for the system chip are supplied from the two regulators. The other devices also operate with the regulated $3.3 \mathrm{~V}$ power supply. The reference current can be determined using an external variable resistor, so that the reference current may be changed. The crystal oscillator generates a $10 \mathrm{MHz}$ clock signal, which is fed to the ASIC, the A/D converters, and the divider through two series inverters. The divider produces a sampling frequency of $400 \mathrm{kHz}$. Six A/D converters operate synchronously with the sampling frequency and output digitized signals of the waveforms. Each bit of the A/D converter output is serial.

Table 3 presents the model number, current, and power loss of all the devices in the 


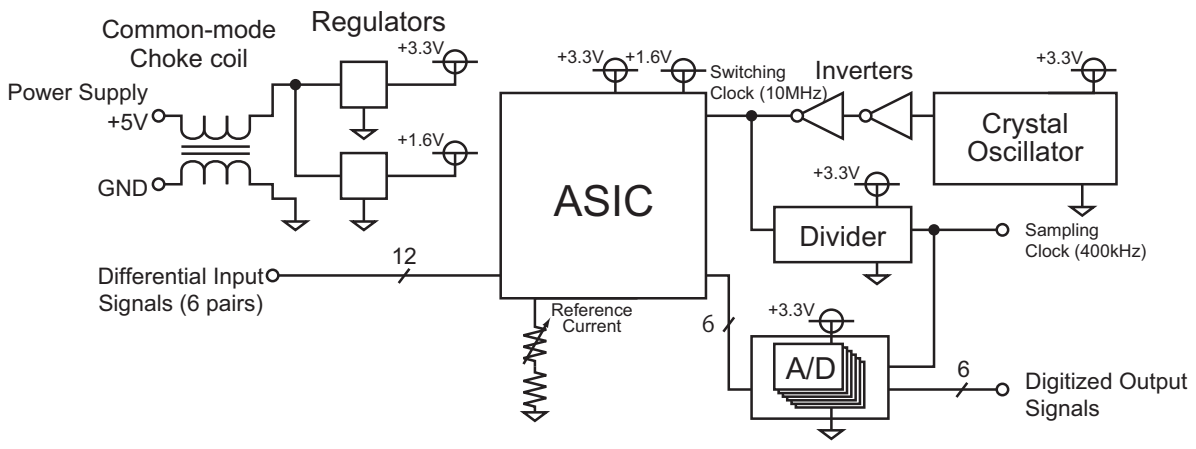

Figure 6. Block diagram of the waveform receiver.

waveform receiver. The regulator for $3.3 \mathrm{~V}$ and the ASIC account for a large portion of the power consumption. Therefore, these two devices should have specified heat radiators for a flight module.

Table 3. Device model, current and power loss of peripheral circuitry.

\begin{tabular}{lccc}
\hline Device & Model Number & Current $[\mathrm{mA}]$ & Power Loss [mW] \\
\hline Common-mode Choke Coil & ACM4532-801-2P & & \\
Regulator (3.3 V) & LM1117 & 94 & 197 \\
Regulator (1.6 V) & KC7050B10.0000C31A00 & & 52 \\
Crystal Oscillator & TC7PA05FU & & 0.09 \\
Inverter & TC74HC40102 & $5.5 \times 6$ & 0.01 \\
Divider & LTC2355I-14 & 50 & $18 \times 6$ \\
A/D Converters & & 105 & 165 \\
\hline ASIC & Total & & 525 \\
\hline
\end{tabular}

\subsection{Overall Evaluation}

Figure 7 shows photographs of the fabricated waveform receiver. The panels (a) and (b) in Figure 7 show the top and back of the printed circuit board, respectively. The system chip is installed on the upper-right corner of the top side. Two 15-pin MDM connectors are used: one for the power lines and input signals, and the other for the output signals. The A/D converters, oscillator, and the divider on the back are separated from the analogue ground plane by the digital ground plane inside the board.

The dimensions of the waveform receiver board are $45 \times 50 \mathrm{~mm}^{2}$. Compared to the LFA presented in Table 1, the area per observation channel of the present device is reduced to less than $1 / 20$. The weight and power consumption of the receiver are 24.9 


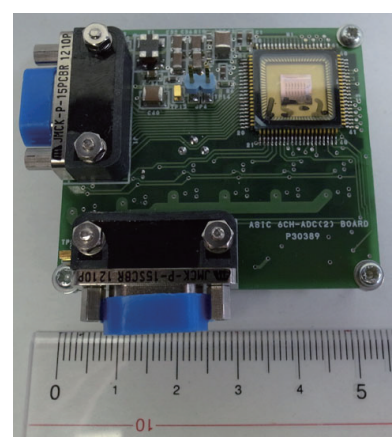

(a) Top side

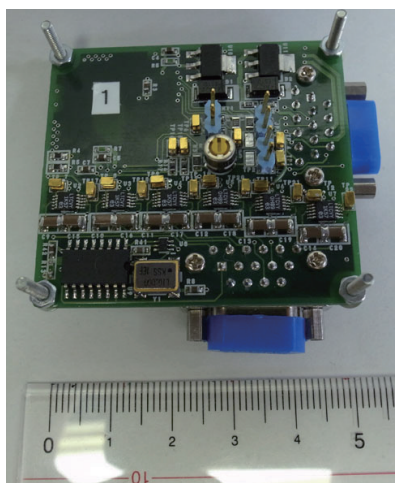

(b) Back side

Figure 7. Photographs of both surfaces of the fabricated waveform receiver.

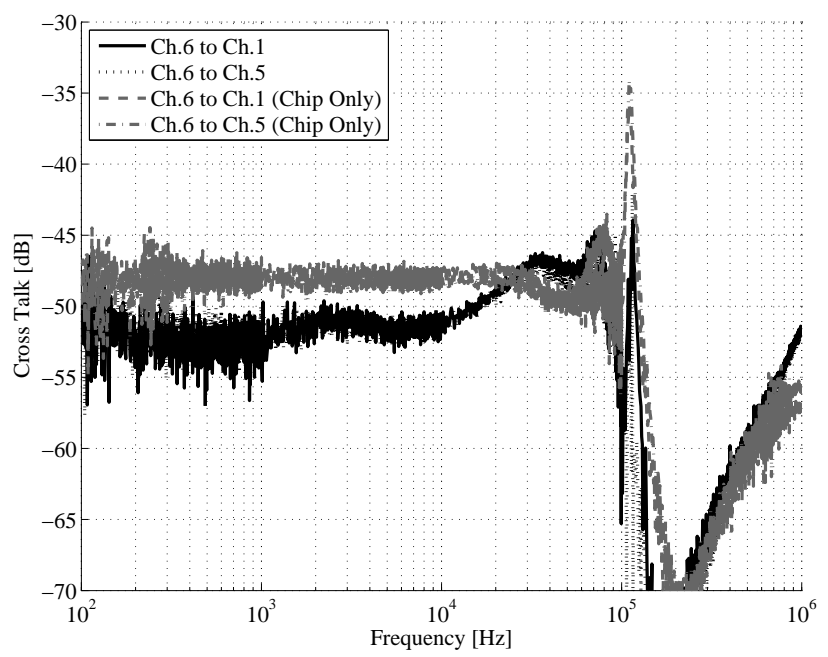

Figure 8. Crosstalk of the waveform receiver and the system chip from channel 6 to channels 1 and 5 .

$\mathrm{g}$ and $525 \mathrm{~mW}$, respectively.

Figure 8 shows the measurement results for crosstalk of the circuit board and system chip. The crosstalk of the system chip is larger than that of the circuit board, because of the coupling in the chip-testing board. Crosstalk is typically required to be less than $-50 \mathrm{~dB}$. In Figure 8, the crosstalk requirement is fulfilled at frequencies lower than $20 \mathrm{kHz}$. Although the crosstalk becomes larger than the typical requirements in the frequency band higher than $20 \mathrm{kHz}$, the crosstalk is acceptable up to $-40 \mathrm{~dB}$, compared to that for the search coil of the on-board BepiColombo/MMO [12].

The sensitivity of the circuit board shown in Figure 9 is calculated from the measured output noise voltage densities at the input of the $\mathrm{A} / \mathrm{D}$ converter and the frequency responses. The sensitivities for the medium- and high-gain configurations are better than that for low gain. The noise is amplified as much as the pass-band gain for the high-gain configuration, so that the sensitivity at high gain is approximately equal 


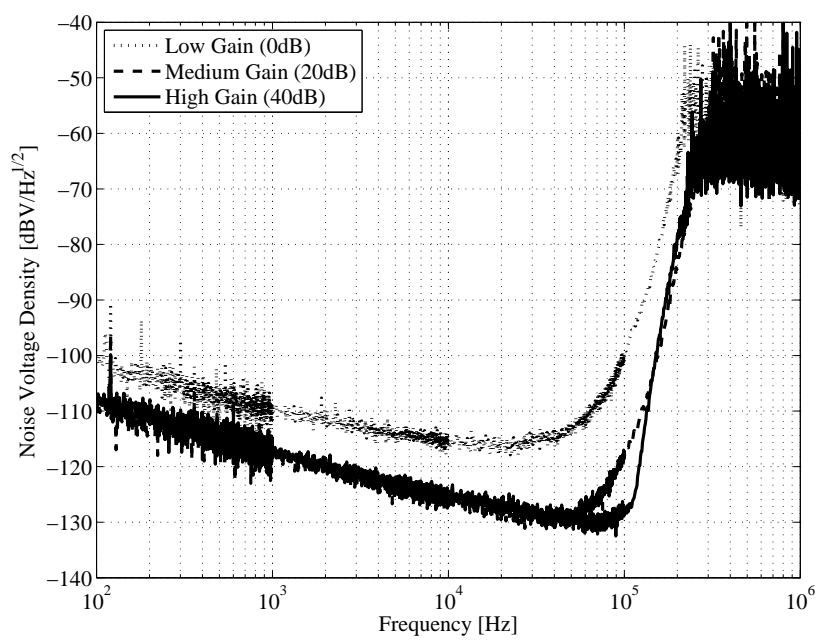

Figure 9. Sensitivity of the circuit board.

to that at medium gain. The sensitivity results indicate that the developed circuit board is suitable for use on scientific missions. However, the $1 / f$ noise in the low frequency band raises considerable concern regarding the sensitivity. To reduce the $1 / f$ noise, a chopper amplifier and correlated double sampling may be suitable.

It is assumed that the waveform receiver will operate in the temperature range from -30 to $60{ }^{\circ} \mathrm{C}$. The correct operation of all components has been confirmed in this range by temperature testing. When the temperature changes from -30 to $60{ }^{\circ} \mathrm{C}$, the change in the gain at $10 \mathrm{kHz}$ is $-0.6,-2.1$, and $-1.8 \mathrm{~dB}$ for the low, medium, and high gain settings, respectively. The change in sensitivity at $10 \mathrm{kHz}$ is $-3.4,+0.7$, and $+0.8 \mathrm{~dB}$ for the respective gain settings. The cut-off frequency changes within $+2 \mathrm{kHz}$. Thus, the developed waveform receiver is compatible with the thermal environment inside a spacecraft.

Radiation tolerance is also an important issue to be investigated, although the waveform receiver presented here has yet to be tested. Single-event effects and total critical ionizing dose are potentially harmful to the A/D converters, inverter and the divider ICs. However, this is outside the scope of the present paper. Radiation-intolerant devices in the peripheral circuitries could be replaced with alternatives that have been confirmed as radiation tolerant, which may require some slight design changes. We have prospects on the radiation tolerance of the system chip as follows. Total ionizing dose and single event latch-up are considered important for radiation tolerance of the analogue components. The total ionizing dose of the ASIC using the TSMC CMOS process has been confirmed to be over 10 krads for space applications [13]. In order to avoid latch-up, every MOSFET device in the system chip has a substrate or well ring connected to the body node of the device to reduce impedance between the source and body nodes of the device. Hence, the system chip, the key component of the waveform receiver, would be expected to be radiation tolerant. However, this should be proved 
experimentally; therefore, radiation tests for the system chip and the waveform receiver are planned for the future.

\section{Conclusions}

Plasma wave observations are very important in the understanding of space electromagnetic phenomena. The waveforms can provide not only amplitude but also phase information of the plasma waves. We have introduced a waveform receiver miniaturized to dimensions of $45 \times 50 \mathrm{~mm}^{2}$, which is half the size of a business card. The design and performance of the developed waveform receiver were described. A system chip that includes the analogue components (six channels of filters and amplifiers) of the waveform receiver was developed. The frequency response, dynamic range, sensitivity, and temperature dependence were discussed. Downsizing with the system chip was demonstrated to be effective to realize an instrument that satisfies the requirements for miniaturization and plasma wave observation. Consequently, a miniaturized instrument is expected to be used on future multi-spacecraft and/or planetary exploration missions.

\section{Acknowledgments}

The present study was supported in part by the VLSI Design and Education Center (VDEC) of the University of Tokyo in collaboration with Cadence Design Systems, Inc. and SiliConsortium Ltd. The mask layout used in the present study was designed in collaboration with Digian Technology Inc. The design and fabrication of the printed circuited board was supported by Meiwa System Corporation. Additional support was provided by a Grant-in-Aid for Japan Society for the Promotion of Science (JSPS) Fellows, Grants-in-Aid for Scientific Research A (19204048, 23244097), and a Japan Aerospace Exploration Agency (JAXA) Grant-in-Aid for Fundamental Development and Experimentation of On-board Instruments. Partial support was also provided by the Yamada Science Foundation.

\section{References}

[1] H Matsumoto, I Nagano, R R Anderson, H Kojima, K Hashimoto, M Tsutsui, T Okada, I Kimura, Y Omura, and M Okada. Plasma wave observations with geotail spacecraft. Journal of geomagnetism and geoelectricity, 46(1):59-95, 1994.

[2] H Matsumoto, H Kojima, T Miyatake, Y Omura, M Okada, I Nagano, and M Tsutsui. Electrostatic solitary waves (esw) in the magnetotail: Ben wave forms observed by geotail. Geophysical Research Letters, 21(25):2915-2918, 1994.

[3] J L Bougeret, M L Kaiser, P J Kellogg, R Manning, K Goetz, S J Monson, N Monge, L Friel, C A Meetre, C Perche, L Sitruk, and S Hoang. Waves: The radio and plasma wave investigation on the wind spacecraft. Space Science Reviews, 71:231-263, 1995. 10.1007/BF00751331.

[4] D A Gurnett, R L Huff, and D L Kirchner. The wide-band plasma wave investigation. Space Science Reviews, 79:195-208, 1997. 10.1023/A:1004966823678. 
[5] J Bonnell, F Mozer, G Delory, A Hull, R Ergun, C Cully, V Angelopoulos, and P Harvey. The electric field instrument (efi) for themis. Space Science Reviews, 141:303-341, 2008. 10.1007/s11214-008-9469-2.

[6] Y Kasahara, Y Goto, K Hashimoto, T Imachi, A Kumamoto, T Ono, and H. Matsumoto. Plasma wave observation using waveform capture in the lunar radar sounder on board the selene spacecraft. Earth, planets and space, 60(4):341-351, 2008.

[7] B Mossawir, I R Linscott, U S Inan, J L Roeder, J V Osborn, S C Witczak, E E King, and S D LaLumondiere. A TID and SEE radiation-hardened, wideband, low-noise amplifier. IEEE Transactions on Nuclear Science, 53(6):3439-3448, 2006.

[8] H Fukuhara, H Kojima, S Okada, H Ikeda, and H Yamakawa. Toward a waveform receiver on a chip dedicated to plasma wave instrument onboard scientific spacecraft. In Aerospace Conference 2011 IEEE, pages 1-9, March 2011.

[9] H Kojima, H Fukuhara, Y Mizuochi, S Yagitani, H Ikeda, Y Miyake, H Usui, H Iwai, Y Takizawa, Y Ueda, and H. Yamakawa. Miniaturization of plasma wave receivers onboard scientific satellites and application to the sensor network system for monitoring electromagnetic environments in space. Advances in Geosciences, 21:461-481, may 2010.

[10] H Matsumoto, T Okada, K Hashimoto, I Nagano, S Yagitani, . Tsutsui, Y Kasaba, K Tsuruda, H Hayakawa, A Matsuoka, S Watanabe, H Ueda, I Kimura, Y Kasahara, Y Omura, T Matsumura, T Imachi, K Ishisaka, and Y. Tateno. Low frequency plasma wave analyzer (lfa) onboard the planet-b spacecraft. Earth Planets Space, 50(3):223-228, 1998.

[11] H Fukuhara, H Kojima, S Okada, H Ikeda, and H. Yamakawa. Temperature compensated gm-c filter for plasma wave receivers onboard scientific spacecraft. IEICE Tranaction on Electronics Japenese Edtion, 94(6):155-162, 2011. (in Japanese).

[12] Y Kasaba, J-L Bougeret, L G Blomberg, H Kojima, S Yagitani, M Moncuquet, J-G Trotignon, G Chanteur, A Kumamoto, Y Kasahara, J Lichtenberger, Y Omura, K Ishisaka, and $\mathrm{H}$ Matsumoto. The plasma wave investigation (PWI) onboard the BepiColombo/MMO: first measurement of electric fields, electromagnetic waves, and radio waves around mercury. Planetary and Space Science, 58:238-278, 2010.

[13] H Nakajima, D Matsuura, N Anabuki, E Miyata, H Tsunemi, J P Doty, H Ikeda, and H Katayama. Development of X-ray CCD camera system with high readout rate using ASIC. Nuclear Instruments and Methods in Physics Research Section A: Accelerators, Spectrometers, Detectors and Associated Equipment, 610(1):78-82, 2009. 